\title{
The Effect of Rabeprazole on Injection Sclerotherapy Complications after First Attack Bleeding Esophageal Varices
}

\author{
Eman El-Shami, Ahmad. S. Sherbini , Ashraf Metwally \\ Tropical Medicine Department, Faculty of Medicine, Zagazig University, Egypt
}

Corresponding Author Ahmad S. Sherbini Mobile:

$+201124811480$

E mail:

ahmadsakr65@yahoo. com

Key words: oesophageal varices, sclerotherapy, proton pump inhibitors (PPI), rabeprazole
Background and study aim : Endoscopic variceal sclerotherapy (EVS) effectively controls bleeding esophageal varices $(\mathrm{OV})$, however it has some adverse effects including sclerosant ulcers, chest pain, dysphagia and odynophagia. Gastric acid plays a central role in mediating and aggravating these complications. Proton pump inhibitors (PPI) are the most potent pharmacologic agents for inhibition of gastric acid secretion. Therefore, these agents are the logical candidates to combat the effects that gastric acid plays in post EVS complications. However, some authors still believe that there is no strong evidence to support their use. This study was designed to evaluate the effects of the use of PPI (rabeprazole) for 8 weeks after endoscopic sclerotherapy for first attack variceal bleeding on the prevention and treatment of complications after EVS. Moreover, we aim to assess the presence of any adverse effects for the use of this drug for this period in this specific patients group. Patients and methods: One hundred patients with first attack variceal bleeding were included in the study. They were allocated randomly into a test group which received $20 \mathrm{mg}$ rabeprazole once daily oral

\section{INTRODUCTION}

Portal hypertension is the leading cause of morbidity and mortality in liver cirrhosis. Complications of portal hypertension in cirrhotic patients include esophageal and gastric varices, portal hypertensive gastropathy, ascites, hepatorenal and hepatopulmonary syndromes as well as portopulmonary hypertension [1]. At the time of diagnosis about $60 \%$ of cirrhotic patients have esophageal varices of different grades. In patients without varices, the rate of developing esophageal varices is about 5\% annually. Acute variceal bleeding is a medical emergency and a life threatening event with a mortality rate of about $25 \%$ [2]. dose following endoscopic sclerotherapy starting 6 hours after injection sclerotherapy and continued for 2 months and a control group which did not receive rabeprazole after sclerotherapy. For both groups, endoscopic, laboratory and clinical data were monitored every two weeks for a period 2 months.

Results: The test group had significantly lower frequency of all post sclerotherapy adverse symptoms, (dysphagia, odynophagia, heart burn, retrosternal and epigastric pain as well as dyspepsia) as well as lower overall rate of re-bleeding (14\% vs $46 \%$ in the control group). There were no significant differences in the hematological parameters or endoscopic findings between test and control groups. Moreover, the use of the drug for two months was not associated with any significant infectious or non infectious complications including fever, hepatic encephalopathy, SBP, diarrhea and chest infection.

Conclusion: Rabeprazole use decreases post-sclerotherapy symptoms and decrease the rate of rebleeding after sclerotherapy without any increasing the complications related to acid supression.

Although $50 \%$ of all esophageal variceal bleeding episodes stop spontaneously, the rebleeding rate is high with about $50 \%$ of patients experiencing a second episode, usually within 2 weeks from the first episode. A second episode of bleeding puts the patient at a high mortality risk and is thus the reason for starting therapy as soon as possible [3]. Endoscopic sclerortherapy should be performed early after hospital admission, assuring that the patient is resuscitated and hemodynamically stable [4]. Endoscopic variceal sclerotherapy (EVS) effectively controls bleeding of esophageal varices $(\mathrm{OV})$, however it has some adverse effects including post injection hemorrhage, chest pain, dysphagia and odynophagia [5]. 
Proton pumps are located on the cytoplasmic membrane of gastric parietal cells. They create an acidic environment in the gastric lumen through exchanging one hydrogen ion for one potassium ion via the hydrogen/potassium adenosine triphosphatase enzyme system (the $\mathrm{H}^{+} / \mathrm{K}^{+}$ATPase pump) [6]. The proton pump is the terminal stage in gastric acid secretion, being directly responsible for secreting $\mathrm{H}^{+}$ions into the gastric lumen, making it an ideal target for inhibiting acid secretion [7]. Proton-pump inhibitors (PPIs) are a group of drugs whose main action is a pronounced and long-lasting reduction of gastric acid production. They act by irreversibly blocking the hydrogen/potassium adenosine triphosphatase enzyme system (the $\mathrm{H}^{+} / \mathrm{K}^{+}$ATPase, or, more commonly, the gastric proton pump) of the gastric parietal cells [6]. PPIs thereby inhibit both basal and stimulated gastric acid secretion, independent of the nature of parietal cell stimulus. They act through blocking acid secretion from all three pathways (neuronal, paracrine and endocrine) simultaneously, so they are considered the most potent medications used to reduce gastric acid secretion [8].

Although all PPIs are effective in treatment of acid-related conditions, there are some differences in their clinical performance, regarding the degree and duration of gastric acid suppression [9]. Differences in PPIs hepatic metabolism may affect both efficacy and consistency, leading to small but significant variation in patient outcomes. PPI selection should therefore involve awareness of these relevant issues [10]. Acid suppressive therapy after EVS is advised as gastric acid may exacerbate post injection ulcers and delay healing [11]. Proton pump inhibitors (PPI) are the most potent pharmacologic agents for inhibition of gastric acid secretion. Therefore, these agents are the logical candidates to control the gastric acid injurious effects on post EVS complications [12]. However, some authors consider its use to be habit related and not evidence based [13].

\section{Aim of the work :}

This study aims to evaluate the effects of rabeprazole administration for 8 weeks after endoscopic sclerotherapy for first attack variceal bleeding on the prevention and treatment of complications after EVS. Moreover, we aim to assess the presence of any adverse effects as a result of the use of this drug for this period in this specific patients group.

\section{PATIENTS AND METHODS}

This prospective randomized clinical trial was conducted in the Intensive Care Unit (ICU), Inpatient and Endoscopy Units of Tropical Medicine Department, Faculty of Medicine Zagazig University, during the period from September 2013 to July 2014.

\section{Inclusion criteria :}

1. Presence of liver cirrhosis, the diagnosis of cirrhosis was based on clinical, biochemical and ultrasonographic findings with Child-Pugh grading (group A and B were only included)

2. First attack of upper GIT bleeding, which was proven by upper GIT endoscopy to be coming from esophageal varices.

3. Signed informed consent.

\section{Exclusion criteria :}

1. Uncooperative patients and those unable to give written informed consent or couldn't return for routine follow up.

2. Endoscopically confirmed pre-existing esophageal ulcers.

3. Ongoing therapy with PPI.

4. Patients with other causes of upper GIT bleeding than esophageal varices.

5. Patients with Child-Pugh grade $\mathrm{C}$.

\section{Randomization :}

Patients were randomly alternatively allocated into 2 groups :

- Group I : (test group) Patients with acute variceal bleeding who received $20 \mathrm{mg}$ rabeprazole once daily oral dose half an hour before breakfast following endoscopic sclerotherapy starting 6 hours after injection sclerotherapy for the varices and continued for 2 months.

- Group II: (control group) Patients with acute variceal bleeding who did not receive any PPIs following endoscopic variceal sclerotherapy during the same period, but received only a bland antacid for 48 hours after each endoscopic sclerotherapy.

All patients in the 2 groups were subjected to:

1. Thorough medical history taking including: age, gender, special habits of medical importance, history of other medical diseases, history of drug intake especially NSAID and anti-acid drugs, past attacks of haematemesis ,amount, colour, presence of melena and blood transfused, history of $\mathrm{HCV}$ or $\mathrm{HBV}$ 
infection, previous or current history of hepatic encephalopathy.

\section{Thorough clinical examination including:}

- General examination focusing on consciousness, vital signs, coloures, signs of liver cell failure \& LL edema.

- Local abdominal examination searching for signs of chronic liver disease and portal hypertension.

\section{3. laboratory investigations:}

- Complete blood picture (CBC)

- Liver function tests including: Total and direct serum bilirubin, serum albumin, Serum
Aspartate amino Transferase (AST) and serum Alanine amino Transferase (ALT).

- Prothrombin time (PT) and international normalized ratio (INR).

- Kidney function tests including blood urea and serum creatinine.

- Viral markers, anti HCV and HBs Ag using third generation ELISA kits.

- Ascetic fluid sample, if possible, was obtained and analyzed physically, biochemically, cellularly and bacteriologically.

4. Child-Pugh classification for all patients into: $\mathrm{A}, \mathrm{B}$, and $\mathrm{C}$ class according the severity of cirrhosis [14]:

\begin{tabular}{|l|c|c|c|}
\hline \multicolumn{1}{|c|}{ Measure } & 1 point & 2 points & 3 points \\
\hline Total bilirubin, $(\mathrm{mg} / \mathrm{dl})$ & $<2$ & $2-3$ & $>3$ \\
\hline Serum albumin, $\mathrm{g} / \mathrm{dl}$ & $>3.5$ & $2.8-3.5$ & $<2.8$ \\
\hline PT (seconds prolonged) & $0-4$ & $4-6$ & $>6$ \\
\hline Ascites & None & Mild & Moderate to Severe \\
\hline Hepatic encephalopathy & None & $\begin{array}{c}\text { Grade I-II (or suppressed } \\
\text { with medication) }\end{array}$ & $\begin{array}{c}\text { Grade III-IV (or } \\
\text { refractory) }\end{array}$ \\
\hline
\end{tabular}

\begin{tabular}{|c|c|c|c|}
\hline Points & Class & One year survival & Two year survival \\
\hline $5-6$ & $\mathrm{~A}$ & $100 \%$ & $85 \%$ \\
\hline $7-9$ & $\mathrm{~B}$ & $81 \%$ & $57 \%$ \\
\hline $10-15$ & $\mathrm{C}$ & $45 \%$ & $35 \%$ \\
\hline
\end{tabular}

\section{Abdominal ultrasonography:}

All the patients were examined using TOSHIBA CAPASEE II device. They were examined according to the standard maneuvers. Liver, spleen, portal vein diameter, and presence of free fluid in the abdomen (ascites) were evaluated. The liver was evaluated and its size was noted. Cirrhotic appearance of the liver was shown by the coarse nodular appearance, increased echogenicity, its shrunken size and prominent caudate lobe [15]. Portal vein diameter was measured at a point of crossing the inferior vena cava. The spleen was evaluated for its length by measuring its bipolar diameter at the left mid-axillary line, it is considered enlarged if it was greater than $13 \mathrm{~cm} \mathrm{[16].}$

\section{Upper gastrointestinal endoscopy:}

Endoscopywas done using end flexible videoendoscope (PENTAX VIDEO unit of endoscopy). The patients were positioned on their left lateral position, with head supported on a small firm pillow to remain in a comfortable neutral position and a bite guard in their mouth. Sedation was received and the tip of endoscope was lubricated and checked for being functioning, regarding image quality, air and water, suction, and tip angulations. Then endoscope was introduced gently and under vision. The OV were shown as tortuous bluish cords running longitudinally within the esophagus and covered with mucosa.

The oesophageal varices were graded into 4 grades according to Thakeb et al. [17].

Grade I: small straight cords of varices continued to lower $1 / 3$ of the esophagus.

Grade II: moderate sized clubbed varices with well-defined areas of normal mucosa between them, forming several distinct vertical cords and confined to lower third of esophagus.

Grade III: gross varices extending into the proximal half of the esophagus, which are so large and tortuous, that normal mucosa may not be visible in between unless the esophagus is fully distended with air. 
Grade IV: varices are like those of grade III but with dilated capillaries on top or in between varices, (varices over varices).

Sclerotherapy was done for the oesophageal varices and total amount of sclerosant material and amount injected in each site were recorded.

Portal hypertensive gastropathy was classified according to the Italian endoscopic club classification validated in 1997 [18] :

- PHG grade I: mild redness and congestive mucosa, no mosaic like pattern.
- PHG grade II: Severe redness and a fine reticular pattern separating the areas of raised edematous mucosa (mosaic like pattern) or fine speckling.

- PHG grade III: Point bleeding + grade II.

Episodes of recurrent bleeding during the follow up period were recorded. The severity of recurrent bleeding was classified according to Cappell and friedel [19] as follows :

\begin{tabular}{|l|l|l|l|}
\hline \multicolumn{1}{|c|}{ Physical signs } & \multicolumn{1}{c|}{ Mild } & \multicolumn{1}{c|}{ Moderate } & \multicolumn{1}{c|}{ Severe } \\
\hline Blood loss & <1 L & 1-2 L & > equal 2 L \\
\hline Blood pressure & Normal & Normal -borderline low & Hypotensive \\
\hline Orthostasis & No & Possible & Likely \\
\hline Tachycardia & None- mild & Moderate & Severe \\
\hline Skin & Warm-wellperfused & Diaphoretic & Cool- cold clamy \\
\hline Respiratory rate & Normal & Normal- slightly decreased & Irregular \\
\hline Urine output & Normal & Diminished & Poor \\
\hline Sensorium & Alert- anxious & Anxious & Confused-drowsy \\
\hline
\end{tabular}

Follow up of the patients by daily morning temperature which was recorded by the patients in a sheet for early prediction of portal bacteraemia and bacterial peritonitis along with other infections as pneumonia and infective diarrhea.

Also the patients were also evaluated according to presence or absence of post-sclerotherapy symptoms including: epigastric pain, heart burn, retrosternal chest pain, dysphagia, dyspepsia, and odynophagea upon discharge and during the follow up visits every two weeks.

Patients used the non selective beta blocker (propranolol) for prevention of recurrent variceal bleeding, starting with $20 \mathrm{mg}$ orally twice daily and increased to maximum tolerated dose or until heart rate reaches 55 beats per minute as recommended by the American association of the study of the liver disease [20].

Then follow up of the patients every 2 weeks and for 2 month by upper GIT endoscopy with commenting on the variceal condition as previous, PHG, bleeding and development of sclerosant ulcer. Also follow up every 2 weeks for 2 months by CBC, and follow up of the patients' adverse symptoms as previous.

Follow up of the patient's physical state for the likely development or improvement of ascites, lower limb edema, jaundice, and hepatic encephalopathy (HE). And follow up for development of diarrhea, chest infection, and abdominal pain and tenderness as indicators for SBP.

Twenty seven patients were lost during the whole follow up period, 19 patients died and 8 patients were lost. Deceased and lost patients were replaced by other patients. Finally at the $8^{\text {th }}$ week 4 patients were lost at each group and were not replaced.

The follow up of the patients was done at the endoscopy unit, ICU and the in-patient department. The telephone was another way of contact with the patients.

\section{Statistical analysis}

Data were checked, entered and analyzed using SPSS version 19 EPI-INFO 6 for data processing and statistics. The quantitative data were presented as mean $(\bar{X})$ and standard deviation and were compared using student $t$ test. The categorical data were presented as number and percentage and were compared using Chi-square test $\left(\mathrm{X}^{2}\right)$. For all above-mentioned statistical tests done, the threshold of significance was fixed at 5\% level (P-value). 


\section{RESULTS}

There were no significant differences between the test group (group I) and the control group (group II) as regards mean age and gender distribution as shown in table (1). Table (2) shows that, there are no statistically significant differences between cases and controls regarding the baseline clinical presentations including ascites, jaundice, hepatic encephalopathy, lower limb edema and fever. There were no statistically significant differences between group I and group II regarding all sonographic data e.g. liver size, portal vein diameter, presence and amount of ascites and spleen size as shown in table (3).

Table (4) shows that, there are no statistically significant baseline differences between cases and controls regarding laboratory parameters including (hemoglobin concentration, total leukocytic count, platelet count, albumin level, total and direct bilirubin levels), ALT, AST, PT, INR, and serum creatinine level. There was also no significant difference between the two groups as regards Child's grade as shown in table (5).

There were no significant differences found between the two groups as regards their preliminary endoscopic findings such as number of OV cords, grade of $\mathrm{OV}$ and risky signs, amount of sclerosant material used in sclerotherapy, grade of portal hypertensive gastropathy and duodenopathy as shown in table (6). While Table (7) shows that, there are no statistically significant differences between cases and controls regarding post sclerotherapy symptoms including (dysphagia, odynophagia, retrosternal pain, epigastric pain, heart burn, and dyspepsia) after first endoscopic sclerotherapy setting.

Table (8) shows that, there are no statistically significant differences between cases and controls regarding all three hematological parameters all through the follow up period.

There were no significant differences between the two studied groups as regards all the endoscopic findings such as risky signs, grade and number of OV cords, amount of sclerosant agents used to secure $\mathrm{OV}$ as well as portal hypertensive gastropathy and duodenopathy and frequency of sclerosant ulcer all through the period of follow up as shown in tables 9 and 10 .

The incidences of post sclerotherapy symptoms such as dysphagia, odynophagia, heart burn, retrosternal and epigastric pain and dyspepsia were significantly lower in the test group than in the controls all through the period of follow up as shown in table (11). There was also significant increase in the rate of moderate severity recurrent bleeding at the second week and at the end of the follow up period as shown in table (11). There were no significant differences between the studied groups as regards incidence of ascites, lower limb edema, jaundice, abdominal tenderness, encephalopathy, fever, chest or urinary tract infections as shown in table (12)

Table (1): Demographic data

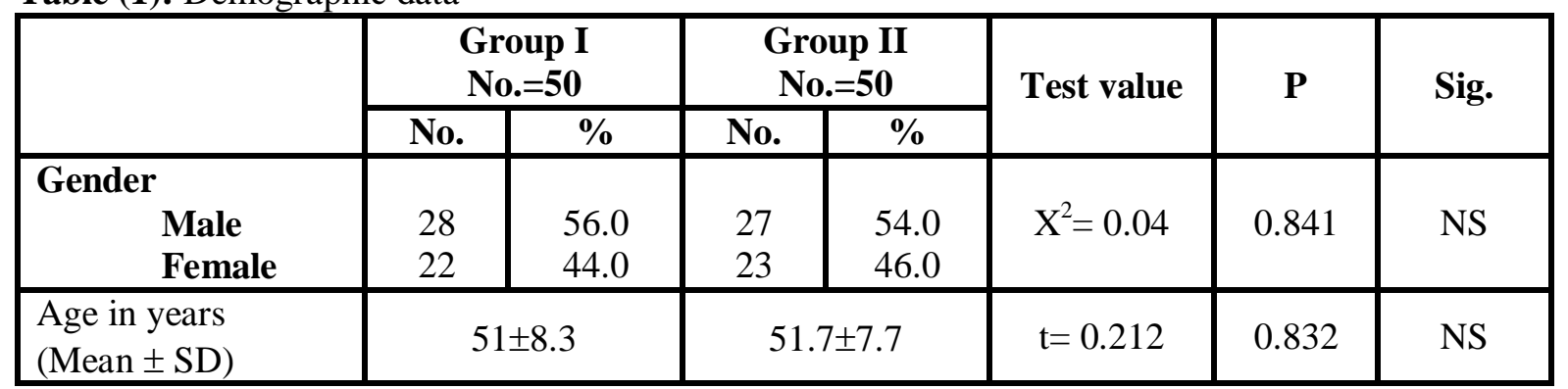

NS: non-significant 
Table (2): Baseline clinical presentations of studied groups

\begin{tabular}{|c|c|c|c|c|c|c|c|c|c|}
\hline & \multicolumn{2}{|c|}{$\begin{array}{c}\text { Group I } \\
\text { No.=50 }\end{array}$} & \multicolumn{2}{|c|}{$\begin{array}{c}\text { Group II } \\
\text { No.=50 }\end{array}$} & \multirow[t]{2}{*}{$\mathrm{X} 2$} & \multirow[t]{2}{*}{$\mathbf{P}$} & \multirow[t]{2}{*}{ Sig. } \\
\hline & & & No. & $\%$ & No. & $\%$ & & & \\
\hline \multicolumn{3}{|l|}{ Ascites } & 18 & 36.0 & 23 & 46.0 & 1.22 & 0.269 & NS \\
\hline \multicolumn{3}{|l|}{ Jaundice } & 6 & 12.0 & 4 & 8.0 & 0.8 & 0.371 & $\mathrm{NS}$ \\
\hline \multicolumn{3}{|l|}{ H E } & 9 & 18.0 & 8 & 16.0 & 0.12 & 0.732 & $\mathrm{NS}$ \\
\hline \multirow[t]{2}{*}{ LL. edema } & \multicolumn{2}{|l|}{ Mild } & 14 & 28.0 & 17 & 34.0 & 0.58 & 0.446 & $\mathrm{NS}$ \\
\hline & \multicolumn{2}{|l|}{ Moderate } & 8 & 16.0 & 10 & 20.0 & 0.44 & 0.505 & $\mathrm{NS}$ \\
\hline \multirow{5}{*}{\multicolumn{2}{|c|}{$\begin{array}{l}\text { Fever } \\
\text { (No. of days of fever over } 38.3 \text { ) }\end{array}$}} & 1 & 4 & 8.0 & 2 & 4.0 & 1.33 & 0.248 & $\mathrm{NS}$ \\
\hline & & 2 & 4 & 8.0 & 4 & 8.0 & 0.25 & 0.617 & $\mathrm{NS}$ \\
\hline & & 3 & 1 & 2.0 & 3 & 6.0 & 2.0 & 0.157 & $\mathrm{NS}$ \\
\hline & & 4 & 1 & 2.0 & 1 & 2.0 & 1.0 & 0.317 & $\mathrm{NS}$ \\
\hline & & 5 & 0 & 0.0 & 1 & 2.0 & 2.0 & 0.157 & $\mathrm{NS}$ \\
\hline
\end{tabular}

Table (3): Comparison of U/S presentation of studied groups

\begin{tabular}{|c|c|c|c|c|c|c|c|c|}
\hline & \multicolumn{2}{|c|}{$\begin{array}{c}\text { Group I } \\
\text { No.=50 }\end{array}$} & \multicolumn{2}{|c|}{$\begin{array}{c}\text { Group II } \\
\text { No.=50 }\end{array}$} & \multirow{2}{*}{$\begin{array}{c}\text { Test } \\
\text { value }\end{array}$} & \multirow[t]{2}{*}{$\mathbf{P}$} & \multirow[t]{2}{*}{ Sig. } \\
\hline & & No & $\%$ & No & $\%$ & & & \\
\hline \multirow[t]{2}{*}{ Liver } & Shrunken & 15 & 30.0 & 10 & 20.0 & $\mathrm{X}^{2}=2.0$ & 0.157 & NS \\
\hline & Average & 35 & 70.0 & 40 & 80.0 & $X^{2}=0.67$ & 0.414 & $\mathrm{NS}$ \\
\hline \multirow{3}{*}{$\begin{array}{l}\text { Portal vein } \\
\text { diameter } \\
(\mathrm{mm})\end{array}$} & $\leq 13 \mathrm{~mm}$ & 2 & 4.0 & 4 & 8.0 & \multirow[t]{2}{*}{$\mathrm{X}^{2}=0.71$} & \multirow[t]{2}{*}{0.399} & \multirow[t]{2}{*}{$\mathrm{NS}$} \\
\hline & $>13 \mathrm{~mm}$ & 48 & 96.0 & 46 & 92.0 & & & \\
\hline & Mean \pm SD & \multicolumn{2}{|c|}{$14.3 \pm 1.3$} & \multicolumn{2}{|c|}{$14.6 \pm 1.5$} & $\mathrm{~T}=0.775$ & 0.44 & NS \\
\hline \multirow[t]{2}{*}{ Ascites } & Present & 22 & 44.0 & 25 & 50.0 & \multirow[t]{2}{*}{$\mathrm{X}^{2}=0.36$} & \multirow[t]{2}{*}{0.548} & NS \\
\hline & Absent & 28 & 56.0 & 25 & 50.0 & & & $\mathrm{NS}$ \\
\hline \multirow[t]{3}{*}{ Spleen ( cm) } & $\leq 13 \mathrm{~mm}$ & 4 & 8.0 & 3 & 6.0 & \multirow[t]{2}{*}{$X^{2}=0.15$} & \multirow[t]{2}{*}{0.695} & \multirow[t]{2}{*}{$\mathrm{NS}$} \\
\hline & $>13 \mathrm{~mm}$ & 46 & 92.0 & 47 & 94.0 & & & \\
\hline & Mean \pm SD & \multicolumn{2}{|c|}{$15 \pm 1.8$} & \multicolumn{2}{|c|}{$14.9 \pm 1.8$} & $\mathrm{~T}=0.173$ & 0.863 & NS \\
\hline
\end{tabular}

Table (4): Comparison of laboratory parameters of studied groups

\begin{tabular}{|l|c|c|c|c|c|}
\hline \multirow{2}{*}{} & $\begin{array}{c}\text { Group I } \\
\text { No.=50 }\end{array}$ & $\begin{array}{c}\text { Group II } \\
\text { No.=50 }\end{array}$ & \multirow{2}{*}{ t } & \multirow{2}{*}{ P } & \multirow{2}{*}{ Sig. } \\
\cline { 2 - 4 } & Mean \pm SD & Mean \pm SD & & & \\
\hline WBC $($ cellX10/ml) & $8 \pm 3.8$ & $8.2 \pm 3.8$ & 0.254 & 0.8 & NS \\
\hline Hb(g/dl) & $7.2 \pm 0.9$ & $7.1 \pm 0.9$ & 0.552 & 0.582 & NS \\
\hline Platelet $(\mathbf{X 1 0} / \mathbf{m l})$ & $85.1 \pm 31.3$ & $88.4 \pm 36.5$ & 0.486 & 0.628 & NS \\
\hline ALT(IU/ml) & $64.5 \pm 19.8$ & $65.2 \pm 29.1$ & 0.145 & 0.885 & NS \\
\hline AST(IU/ml) & $83.9 \pm 27.9$ & $87.8 \pm 45.1$ & 0.512 & 0.61 & NS \\
\hline Total bilirubin (mg/dl) & $2.1 \pm 1.2$ & $1.9 \pm 1.1$ & 0.75 & 0.387 & NS \\
\hline Direct bilirubin (mg/dl) & $0.7 \pm 0.3$ & $0.65 \pm 0.3$ & 0.243 & 0.808 & NS \\
\hline Albumin $(\mathbf{g} / \mathbf{d l})$ & $3.1 \pm 0.3$ & $3 \pm 0.3$ & 0.314 & 0.754 & NS \\
\hline INR & $1.4 \pm 0.3$ & $1.4 \pm 0.2$ & 0.0 & 1.0 & NS \\
\hline PT(second) & $17.2 \pm 2.3$ & $17.4 \pm 2$ & 0.518 & 0.606 & NS \\
\hline Creatinine (mg/dl) & $1 \pm 0.5$ & $1 \pm 0.4$ & 0.195 & 0.846 & NS \\
\hline
\end{tabular}


Table (5): Comparison of Child Pugh grade of studied groups

\begin{tabular}{|c|c|c|c|c|c|c|c|}
\hline & \multicolumn{2}{|c|}{$\begin{array}{c}\text { Group I } \\
\text { No.=50 }\end{array}$} & \multicolumn{2}{|c|}{$\begin{array}{c}\text { Group II } \\
\text { No.=50 }\end{array}$} & \multirow{2}{*}{ X2 } & \multirow{2}{*}{ P } & Sig. \\
\cline { 2 - 5 } & No. & \% & No. & \% & & & NS \\
\hline A & 22 & 44.0 & 20 & 40.0 & 0.19 & 0.663 & NS \\
\hline B & 28 & 56.0 & 30 & 60.0 & 0.14 & 0.71 & \\
\hline
\end{tabular}

Table (6): Upper GIT endoscopy presentation of group I and group II

\begin{tabular}{|c|c|c|c|c|c|c|c|c|}
\hline & \multicolumn{2}{|c|}{$\begin{array}{l}\text { Group I } \\
\text { No.=50 }\end{array}$} & \multicolumn{2}{|c|}{$\begin{array}{c}\text { Group II } \\
\text { No. }=\mathbf{5 0}\end{array}$} & \multirow[t]{2}{*}{$\mathbf{X} 2$} & \multirow[t]{2}{*}{$\mathbf{P}$} & \multirow[t]{2}{*}{ Sig. } \\
\hline & & No. & $\%$ & No. & $\%$ & & & \\
\hline Risky signs & Present & 20 & 40.0 & 24 & 48.0 & 0.73 & 0.394 & NS \\
\hline \multirow{3}{*}{$\begin{array}{l}\text { No. of Oesophageal } \\
\text { varices cords }\end{array}$} & 2 & 18 & 36.0 & 17 & 34.0 & 0.06 & 0.811 & $\mathrm{NS}$ \\
\hline & 3 & 27 & 54.0 & 27 & 54.0 & 0.04 & 0.847 & $\overline{N S}$ \\
\hline & 4 & 5 & 10.0 & 6 & 12.0 & 0.18 & 0.669 & $\mathrm{NS}$ \\
\hline \multirow{4}{*}{$\begin{array}{l}\text { Oesophageal varices } \\
\text { (OV) grade }\end{array}$} & $\mathrm{I}$ & 2 & 4.0 & 0 & 0.0 & \multirow{4}{*}{2.17} & \multirow{4}{*}{0.538} & \multirow{4}{*}{ NS } \\
\hline & II & 16 & 32.0 & 15 & 30.0 & & & \\
\hline & III & 24 & 48.0 & 26 & 52.0 & & & \\
\hline & IV & 8 & 16.0 & 9 & 18.0 & & & \\
\hline \multicolumn{2}{|c|}{ Amount of EO ( Mean \pm SD) } & \multicolumn{2}{|c|}{$10.2 \pm 4.3$} & \multicolumn{2}{|c|}{$10.9 \pm 5$} & $t=0.752$ & 0.454 & NS \\
\hline \multirow[t]{3}{*}{ PHG grade } & $\mathrm{I}$ & 4 & 8.0 & 3 & 6.0 & 0.29 & 0.593 & $\mathrm{NS}$ \\
\hline & II & 23 & 46.0 & 22 & 44.0 & 0.04 & 0.833 & $\mathrm{NS}$ \\
\hline & III & 23 & 46.0 & 25 & 50.0 & 0.16 & 0.689 & NS \\
\hline \multicolumn{2}{|l|}{ Duodenopathy } & 16 & 32.0 & 18 & 36.0 & 0.24 & 0.628 & NS \\
\hline
\end{tabular}

Table (7): Post sclerotherapy symptoms of studied groups after first injection sclerotherapy

\begin{tabular}{|l|c|c|c|c|c|c|c|}
\hline \multirow{2}{*}{} & \multicolumn{2}{|c|}{$\begin{array}{c}\text { Group I } \\
\text { No.=50 }\end{array}$} & \multicolumn{2}{c|}{$\begin{array}{c}\text { Group II } \\
\text { No.=50 }\end{array}$} & \multirow{2}{*}{ X2 } & \multirow{2}{*}{ P } & \multirow{2}{*}{ Sig. } \\
\cline { 2 - 5 } & No. & $\mathbf{\%}$ & No. & \% & & & \\
\hline Dysphagia & 30 & 60 & 32 & 64 & 0.17 & 0.68 & NS \\
\hline Epigastric pain & 43 & 86.0 & 47 & 94.0 & 1.78 & 0.182 & NS \\
\hline Heart burn & 40 & 80.0 & 38 & 76.0 & 0.23 & 0.629 & NS \\
\hline Odynophagia & 29 & 58.0 & 33 & 66.0 & 0.68 & 0.409 & NS \\
\hline Retrosternal pain & 47 & 94.0 & 43 & 86.0 & 1.78 & 0.182 & NS \\
\hline Dyspepsia & 40 & 80.0 & 45 & 90.0 & 1.96 & 0.161 & NS \\
\hline
\end{tabular}


Table (8): CBC of group I and group II after 2 weeks follow up

\begin{tabular}{|c|c|c|c|c|c|c|}
\hline & $\begin{array}{l}\text { Group I } \\
\text { No. }=50\end{array}$ & $\begin{array}{c}\text { Group II } \\
\text { No. }=50\end{array}$ & $\mathbf{T}$ & $\mathbf{P}$ & Sig \\
\hline \multirow{3}{*}{ 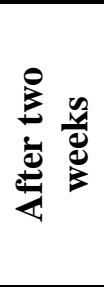 } & $\begin{array}{l}\mathrm{WBC}\left(\mathrm{X}^{3} 0^{3} / \mathrm{ml}\right) \\
\text { Mean } \pm \text { SD }\end{array}$ & $5.5 \pm 1.9$ & $5.6 \pm 2.2$ & 0.137 & 0.891 & NS \\
\hline & $\begin{array}{l}\text { Hb }(\mathrm{g} / \mathrm{dl}) \\
\text { Mean } \pm \text { SD }\end{array}$ & $9.3 \pm 1$ & $8.8 \pm 2.2$ & 1.331 & 0.186 & NS \\
\hline & $\begin{array}{l}\text { Platelet }\left(\mathrm{X} 10^{3} / \mathrm{ml}\right) \\
\text { Mean } \pm \text { SD }\end{array}$ & $83.3 \pm 21.5$ & $80.4 \pm 27.2$ & 0.583 & 0.562 & NS \\
\hline \multirow{3}{*}{ 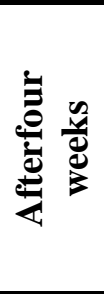 } & $\begin{array}{l}\mathrm{WBC}(\mathrm{X10} / \mathrm{ml}) \\
\text { Mean } \pm \text { SD }\end{array}$ & $7.1 \pm 2.3$ & $6.9 \pm 2.6$ & 0.17 & 0.685 & NS \\
\hline & $\begin{array}{l}\text { Hb }(\mathrm{g} / \mathrm{dl}) \\
\text { Mean } \pm \mathrm{SD}\end{array}$ & $9.5 \pm 1$ & $9.1 \pm 1.5$ & 2.46 & 0.119 & NS \\
\hline & $\begin{array}{l}\text { Platelet }\left(\mathrm{X10}{ }^{3} / \mathrm{ml}\right) \\
\text { Mean } \pm \text { SD }\end{array}$ & $81.6 \pm 22.9$ & $82.5 \pm 25.1$ & 0.172 & 0.864 & NS \\
\hline \multirow{3}{*}{ 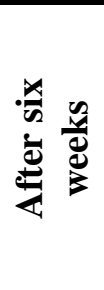 } & $\begin{array}{l}\mathrm{WBC}(\mathrm{X10} / \mathrm{ml}) \\
\text { Mean } \pm \text { SD }\end{array}$ & $7.4 \pm 2$ & $6.9 \pm 2.5$ & 1.22 & 0.272 & NS \\
\hline & $\begin{array}{l}\text { Hb }(\mathrm{g} / \mathrm{dl}) \\
\text { Mean } \pm \mathrm{SD}\end{array}$ & $10.2 \pm 4$ & $9.1 \pm 1.2$ & 3.47 & 0.065 & NS \\
\hline & $\begin{array}{l}\text { Platelet }\left(\mathrm{X10}^{3} / \mathrm{ml}\right) \\
\text { Mean } \pm \text { SD }\end{array}$ & $79.2 \pm 20.7$ & $80.9 \pm 22.6$ & 0.379 & 0.706 & NS \\
\hline \multirow{3}{*}{ 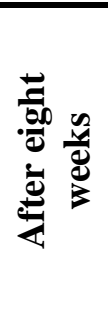 } & $\begin{array}{l}\mathrm{WBC}(\mathrm{X10} / \mathrm{ml}) \\
\text { Mean } \pm \mathrm{SD}\end{array}$ & $8.4 \pm 4.5$ & $7.1 \pm 2.2$ & 3.37 & 0.069 & NS \\
\hline & $\begin{array}{l}\text { Hb }(g / d l) \\
\text { Mean } \pm \text { SD }\end{array}$ & $10 \pm 5$ & $8.4 \pm 5.9$ & 2.14 & 0.147 & NS \\
\hline & $\begin{array}{l}\text { Platelet }\left(\mathrm{X10}^{3} / \mathrm{ml}\right) \\
\text { Mean } \pm \text { SD }\end{array}$ & $79.8 \pm 23.9$ & $80.4 \pm 25.2$ & 0.131 & 0.896 & NS \\
\hline
\end{tabular}


Table (9): Endoscopic findings in studied groups at two and four weeks of follow up

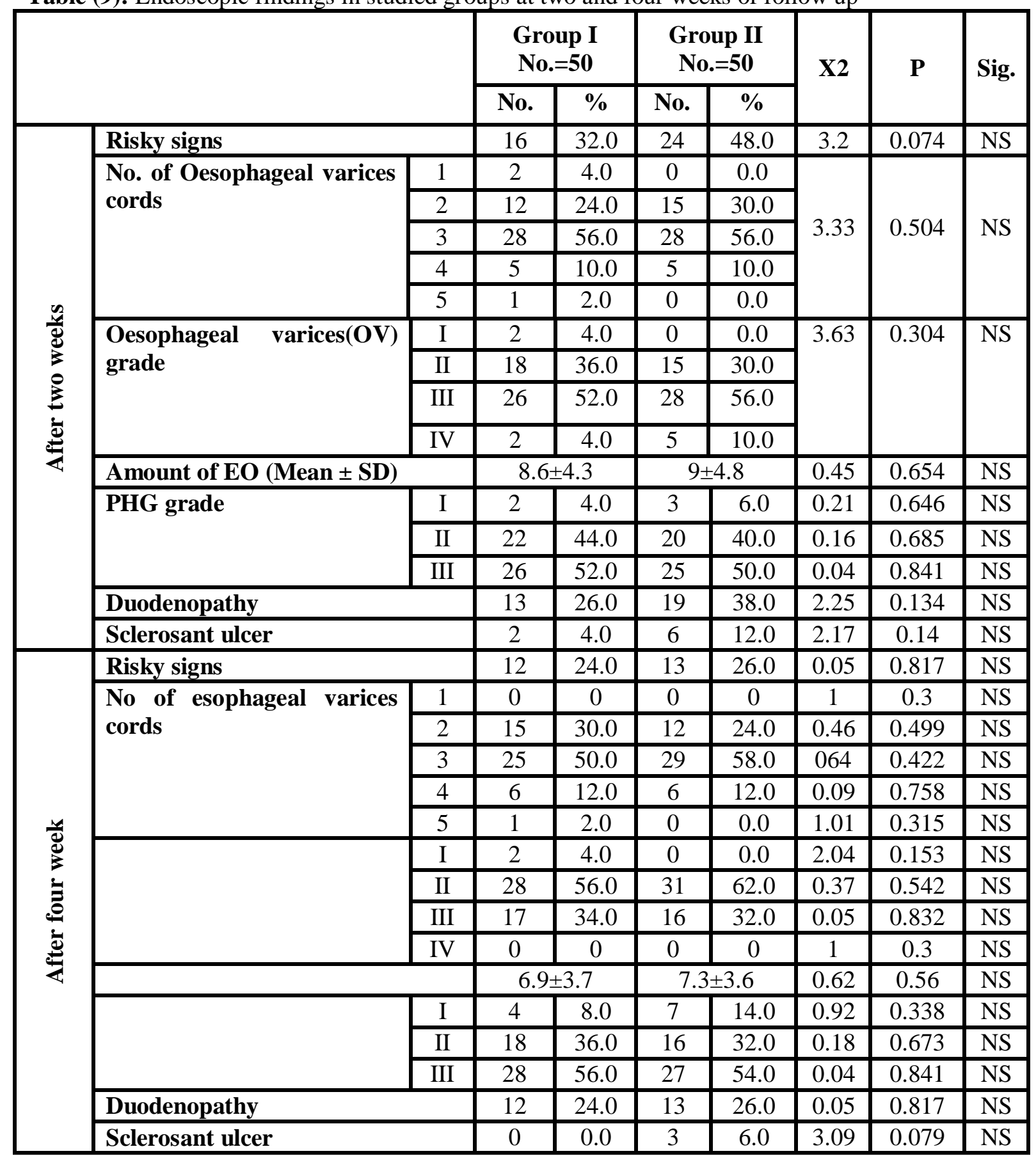


Table (10): Endoscopic findings in studied groups at six weeks and end of follow up

\begin{tabular}{|c|c|c|c|c|c|c|c|c|c|}
\hline & \multicolumn{2}{|c|}{$\begin{array}{l}\text { Group I } \\
\text { No.=50 }\end{array}$} & \multicolumn{2}{|c|}{$\begin{array}{c}\text { Group II } \\
\text { No. }=\mathbf{5 0}\end{array}$} & \multirow[t]{2}{*}{$\mathrm{X} 2$} & \multirow[t]{2}{*}{$\mathbf{P}$} & \multirow[t]{2}{*}{ Sig. } \\
\hline & & & No & $\%$ & No & $\%$ & & & \\
\hline \multirow{16}{*}{ 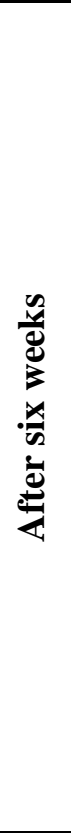 } & \multicolumn{2}{|l|}{ Risky signs } & 8 & 16.0 & 10 & 20.0 & 0.27 & 0.603 & $\mathrm{NS}$ \\
\hline & \multirow{5}{*}{$\begin{array}{l}\text { No. of Oesophageal } \\
\text { varices cords }\end{array}$} & 1 & 3 & 6.0 & 1 & 2.0 & 1.04 & 0.307 & $\mathrm{NS}$ \\
\hline & & 2 & 18 & 36.0 & 13 & 26.0 & 1.17 & 0.279 & NS \\
\hline & & 3 & 19 & 38.0 & 26 & 52.0 & 1.98 & 0.159 & NS \\
\hline & & 4 & 6 & 12.0 & 6 & 12.0 & 0.09 & 0.758 & $\mathrm{NS}$ \\
\hline & & 5 & 1 & 2.0 & 0 & 0.0 & 1.01 & 0.315 & $\mathrm{NS}$ \\
\hline & \multirow{4}{*}{$\begin{array}{l}\text { Oesophageal } \\
\text { varices }(\mathrm{OV}) \text { grade }\end{array}$} & $\bar{I}$ & 7 & $\overline{14.0}$ & 6 & 12.0 & 0.09 & 0.766 & NS \\
\hline & & II & 33 & $\overline{66.0}$ & 30 & 60.0 & 0.39 & 0.534 & NS \\
\hline & & III & 5 & 10.0 & 9 & 18.0 & 1.33 & 0.249 & $\mathrm{NS}$ \\
\hline & & IV & 1 & 2.0 & 1 & 2.0 & 1.0 & 0.317 & NS \\
\hline & \multicolumn{2}{|c|}{ Amount of EO (Mean \pm SD) } & \multicolumn{2}{|c|}{$5.9 \pm 4.1$} & \multicolumn{2}{|c|}{$6.5 \pm 4.1$} & 0.674 & 0.502 & $\mathrm{NS}$ \\
\hline & \multirow[t]{3}{*}{ PHG grade } & $\mathrm{I}$ & 5 & 10.0 & 6 & 12.0 & 0.1 & 0.749 & NS \\
\hline & & II & 18 & 36.0 & 18 & 36.0 & 0.04 & 0.835 & $\mathrm{NS}$ \\
\hline & & III & 27 & 54.0 & 26 & 52.0 & 0.04 & 0.841 & $\mathrm{NS}$ \\
\hline & \multicolumn{2}{|l|}{ Duodenopathy } & 10 & 20.0 & 13 & 26.0 & 0.51 & 0.476 & $\mathrm{NS}$ \\
\hline & \multicolumn{2}{|l|}{ Sclerosant ulcer } & 1 & 2.0 & 5 & 10.0 & 2.84 & 0.092 & $\mathrm{NS}$ \\
\hline \multirow{16}{*}{ 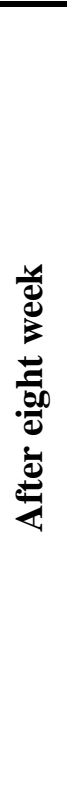 } & \multicolumn{2}{|l|}{ Risky signs } & 6 & $\overline{12.0}$ & 8 & 16.0 & 0.33 & 0.564 & NS \\
\hline & \multirow{5}{*}{$\begin{array}{l}\text { No of esophageal } \\
\text { varices cords }\end{array}$} & 1 & 4 & 8.0 & 4 & 8.0 & 0.14 & 0.712 & $\mathrm{NS}$ \\
\hline & & 2 & 25 & 50.0 & 22 & 44.0 & 0.36 & 0.548 & $\mathrm{NS}$ \\
\hline & & 3 & 13 & 26.0 & 14 & 28.0 & 0.05 & 0.821 & NS \\
\hline & & 4 & 5 & 10.0 & 6 & 12.0 & 0.1 & 0.749 & $\mathrm{NS}$ \\
\hline & & 5 & 3 & 6 & 5 & 10 & 0.54 & 0.461 & $\mathrm{NS}$ \\
\hline & \multirow{4}{*}{$\begin{array}{l}\text { Oesophageal } \\
\text { varices }(\mathrm{OV}) \text { grade }\end{array}$} & I & 5 & $\overline{10.0}$ & 3 & 6.0 & 0.54 & 0.461 & NS \\
\hline & & II & 32 & 64.0 & 33 & 66.0 & 0.1 & 0.749 & NS \\
\hline & & III & 6 & 12.0 & 5 & 10.0 & 0.1 & 0.749 & NS \\
\hline & & $\mathrm{IV}$ & 0 & 0.0 & 0 & 0.0 & & & \\
\hline & \multicolumn{2}{|c|}{ Amount of EO (Mean \pm SD) } & \multicolumn{2}{|c|}{$4.6 \pm 2.7$} & \multicolumn{2}{|c|}{$5 \pm 3$} & 0.49 & 0.485 & NS \\
\hline & \multirow[t]{3}{*}{ PHG grade } & $\mathrm{I}$ & 6 & 12.0 & 5 & 10.0 & 0.1 & 0.749 & $\mathrm{NS}$ \\
\hline & & II & 14 & 28.0 & 19 & 38.0 & 1.13 & 0.288 & $\mathrm{NS}$ \\
\hline & & III & 30 & 60.0 & 26 & 52.0 & 0.65 & 0.42 & $\mathrm{NS}$ \\
\hline & \multicolumn{2}{|l|}{ Duodenopathy } & 8 & 16.0 & 10 & 20.0 & 0.27 & 0.603 & NS \\
\hline & \multicolumn{2}{|l|}{ Sclerosant ulcer } & 0 & 0.0 & 2 & 4.0 & 2.04 & 0.153 & $\mathrm{NS}$ \\
\hline
\end{tabular}


Table (11): Post sclerotherapy symptoms studied groups all through follow up period

\begin{tabular}{|c|c|c|c|c|c|c|c|c|c|}
\hline & \multicolumn{2}{|c|}{$\begin{array}{l}\text { Group I } \\
(\mathrm{No}=\mathbf{5 0})\end{array}$} & \multicolumn{2}{|c|}{$\begin{array}{l}\text { Group II } \\
(\mathrm{No}=50)\end{array}$} & \multirow[t]{2}{*}{$\mathbf{X}^{2}$} & \multirow[t]{2}{*}{$\mathbf{P}$} & \multirow[t]{2}{*}{ Sig. } \\
\hline & & & No & $\%$ & No & $\%$ & & & \\
\hline \multirow{10}{*}{ 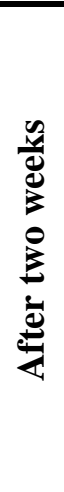 } & \multicolumn{2}{|l|}{ Dysphagia } & 20 & 40 & 40 & 80 & 16.67 & $<0.001$ & $\mathrm{HS}$ \\
\hline & \multicolumn{2}{|l|}{ Epigastric pain } & 21 & 42 & 43 & 86 & 21.01 & $<0.001$ & $\mathrm{HS}$ \\
\hline & \multicolumn{2}{|l|}{ Heart burn } & 18 & 36 & 40 & 80 & 19.87 & $<0.001$ & $\overline{\mathrm{HS}}$ \\
\hline & \multicolumn{2}{|l|}{ Odynophagia } & 13 & 26 & 27 & 54 & 8.17 & 0.004 & $\mathrm{~S}$ \\
\hline & \multicolumn{2}{|l|}{ Retrosternal pain } & 12 & 24 & 40 & 80 & 31.41 & $<0.001$ & $\overline{\mathrm{HS}}$ \\
\hline & \multicolumn{2}{|c|}{ Dyspepsia } & 18 & 36 & 39 & 78 & 17.99 & $<0.001$ & $\mathrm{HS}$ \\
\hline & \multirow{4}{*}{$\begin{array}{l}\text { Recurrent } \\
\text { bleeding }\end{array}$} & Mild & 2 & 4 & 0 & 0 & 2.04 & 0.153 & NS \\
\hline & & Moderate & 1 & 2 & 7 & 14 & 4.89 & 0.027 & $\mathrm{~S}$ \\
\hline & & Severe & 0 & 0 & 1 & 2 & 1.01 & 0.315 & $\mathrm{NS}$ \\
\hline & & Total & 3 & 6 & 8 & 16 & 2.55 & 0.11 & $\mathrm{NS}$ \\
\hline \multirow{10}{*}{ 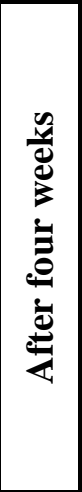 } & \multicolumn{2}{|l|}{ Dysphagia } & 13 & 26 & 30 & 60 & 11.79 & $<0.001$ & $\mathrm{HS}$ \\
\hline & \multicolumn{2}{|l|}{ Epigastric pain } & 11 & 22 & 33 & 66 & 1.64 & $<0.001$ & $\mathrm{HS}$ \\
\hline & \multicolumn{2}{|l|}{ Heart burn } & 10 & 20 & 24 & 48 & 8.73 & 0.003 & $\mathrm{~S}$ \\
\hline & \multicolumn{2}{|l|}{ Odynophagia } & 3 & 6 & 15 & 30 & 9.76 & 0.002 & $\mathrm{~S}$ \\
\hline & \multicolumn{2}{|c|}{ Retrosternal pain } & 10 & 20 & 30 & 60 & 16.67 & $<0.001$ & $\overline{\mathrm{HS}}$ \\
\hline & \multicolumn{2}{|c|}{ Dyspepsia } & 14 & 28 & 39 & 78 & 25.09 & $<0.001$ & $\mathrm{HS}$ \\
\hline & \multirow{4}{*}{$\begin{array}{l}\text { Recurrent } \\
\text { bleeding }\end{array}$} & Mild & 1 & 2 & 0 & 0 & 1.01 & 0.315 & $\mathrm{NS}$ \\
\hline & & Moderate & 2 & 4 & 3 & 6 & 0.21 & 0.646 & $\mathrm{NS}$ \\
\hline & & Severe & 0 & 0 & 2 & 4 & 2.04 & 0.153 & $\mathrm{NS}$ \\
\hline & & Total & 3 & 6 & 5 & 10 & 0.54 & 0.461 & $\overline{\mathrm{NS}}$ \\
\hline & \multicolumn{2}{|l|}{ Dysphagia } & 10 & 20 & 24 & 48 & 8.73 & 0.003 & $\mathrm{~S}$ \\
\hline & \multicolumn{2}{|l|}{ Epigastric pain } & 11 & 22 & 27 & 54 & 10.87 & $<0.001$ & $\overline{\mathrm{HS}}$ \\
\hline & Heart burn & & 6 & 12 & 14 & 28 & 4 & 0.046 & $\mathrm{~S}$ \\
\hline$\frac{\pi}{2}$ & Odynophagia & & 6 & 12 & 14 & 28 & 4 & 0.046 & $\mathrm{~S}$ \\
\hline$\frac{3}{x}$ & Retrosternal pai & & 13 & 26 & 30 & 60 & 11.79 & $<0.001$ & $\mathrm{HS}$ \\
\hline कै & Dyspepsia & & 13 & 26 & 34 & 68 & 17.7 & $<0.001$ & $\mathrm{HS}$ \\
\hline 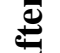 & Recurrent & Mild & 1 & 2 & 1 & 2 & 0.51 & 0.475 & $\mathrm{NS}$ \\
\hline & bleeding & Moderate & 0 & 0 & 5 & 10 & 5.26 & 0.022 & $\mathrm{~S}$ \\
\hline & & Severe & 0 & 0 & 0 & 0 & 1 & 0.31 & $\mathrm{NS}$ \\
\hline & & Total & 1 & 2 & 6 & 12 & 3.84 & 0.049 & $\mathrm{~S}$ \\
\hline & Dysphagia & & 4 & 8 & 12 & 36 & 4.76 & 0.029 & $\mathrm{~S}$ \\
\hline & Epigastric pain & & 10 & 20 & 27 & 54 & 12.4 & $<0.001$ & $\overline{\mathrm{HS}}$ \\
\hline$\frac{n}{0}$ & Heart burn & & 6 & 12 & 15 & 30 & 25.0 & $<0.001$ & $\mathrm{HS}$ \\
\hline 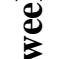 & Odynophagia & & 6 & 12 & 14 & 28 & 4.0 & 0.046 & $\mathrm{~S}$ \\
\hline$\equiv$ & Retrosternal pai & & 10 & 20 & 30 & 60 & 16.67 & $<0.001$ & $\mathrm{HS}$ \\
\hline.$\frac{500}{0}$ & Dyspepsia & & 9 & 18 & 30 & 60 & 18.54 & $<0.001$ & $\mathrm{HS}$ \\
\hline 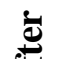 & Recurrent & Mild & 0 & 0 & 4 & 8 & 4.17 & 0.041 & $\mathrm{~S}$ \\
\hline$\sum$ & bleeding & Moderate & 0 & 0 & 0 & 0 & 1 & 0.3 & $\mathrm{NS}$ \\
\hline & & Severe & $\overline{0}$ & 0 & 0 & $\overline{0}$ & 1 & 0.3 & $\overline{N S}$ \\
\hline & & Total & 0 & 0 & 4 & 8 & 4.17 & 0.041 & $\mathrm{~S}$ \\
\hline
\end{tabular}


Table (12): clinical presentation of both studied groups at the end of follow up period

\begin{tabular}{|c|c|c|c|c|c|c|c|c|c|}
\hline & \multicolumn{2}{|c|}{$\begin{array}{l}\text { Group I } \\
(\mathrm{No}=50)\end{array}$} & \multicolumn{2}{|c|}{$\begin{array}{l}\text { Group II } \\
(\mathrm{No}=50)\end{array}$} & \multirow{2}{*}{$\mathbf{X}^{2}$} & \multirow[t]{2}{*}{$\mathrm{P}$} & \multirow{2}{*}{ Sig. } \\
\hline & & & $\overline{\text { No }}$ & $\%$ & $\overline{\text { No }}$ & $\%$ & & & \\
\hline \multirow{5}{*}{\multicolumn{2}{|c|}{$\begin{array}{l}\text { Fever } \\
\text { (No. of days of fever over 38.3) }\end{array}$}} & 1 & 11 & 22.0 & 12 & 24.0 & 0.06 & 0.812 & $\mathrm{NS}$ \\
\hline & & 2 & 8 & 16.0 & 6 & 12.0 & 0.33 & 0.564 & $\mathrm{NS}$ \\
\hline & & 3 & 4 & 8.0 & 7 & 14.0 & 0.92 & 0.338 & NS \\
\hline & & 4 & 5 & 10.0 & 8 & 16.0 & 0.8 & 0.372 & $\mathrm{NS}$ \\
\hline & & 5 & 1 & 2.0 & 3 & 6.0 & 1.04 & 0.307 & $\mathrm{NS}$ \\
\hline \multicolumn{3}{|c|}{ Heptic encephalopathy } & 16 & 32.0 & 13 & 26.0 & 0.44 & 0.509 & $\mathrm{NS}$ \\
\hline \multirow[t]{2}{*}{ Ascites } & \multicolumn{2}{|l|}{ Present } & 27 & 54.0 & 30 & 60.0 & \multirow{2}{*}{0.37} & \multirow{2}{*}{0.545} & \multirow{2}{*}{ NS } \\
\hline & \multicolumn{2}{|l|}{ Absent } & 23 & 46.0 & 20 & 40.0 & & & \\
\hline \multirow[t]{2}{*}{ Jaundice } & \multicolumn{2}{|c|}{ Tinge } & 7 & 14.0 & 3 & 6.0 & 1.78 & 0.182 & $\mathrm{NS}$ \\
\hline & \multicolumn{2}{|c|}{ Clinically apparent } & 3 & 6.0 & 6 & 12.0 & 1.1 & 0.295 & $\mathrm{NS}$ \\
\hline \multirow[t]{2}{*}{ LL oedema } & \multicolumn{2}{|c|}{ Mild } & 13 & 26.0 & 15 & 30.0 & 0.2 & 0.656 & $\mathrm{NS}$ \\
\hline & \multicolumn{2}{|l|}{ Moderate } & 5 & 10.0 & 9 & 18.0 & 1.33 & 0.249 & NS \\
\hline \multicolumn{3}{|c|}{ Abdominal tenderness } & 8 & 16.0 & 6 & 12.0 & 0.33 & 0.564 & $\mathrm{NS}$ \\
\hline \multirow{3}{*}{\multicolumn{2}{|c|}{$\begin{array}{l}\text { Diarrhea } \\
\text { (No. of days of diarrhea) }\end{array}$}} & 1 & 9 & 18.0 & 8 & 16.0 & 0.07 & 0.79 & $\mathrm{NS}$ \\
\hline & & 2 & 7 & 14.0 & 3 & 6.0 & 1.78 & 0.182 & $\mathrm{NS}$ \\
\hline & & 3 & 2 & 4.0 & 1 & 2.0 & 0.34 & 0.558 & $\mathrm{NS}$ \\
\hline \multirow{2}{*}{\multicolumn{2}{|c|}{$\begin{array}{l}\text { Chest infection } \\
\text { (No. of times of chest infection) }\end{array}$}} & 1 & 7 & 14.0 & 4 & 8.0 & 0.92 & 0.338 & $\overline{\mathrm{NS}}$ \\
\hline & & 2 & 1 & 2.0 & 0 & $\overline{0.0}$ & $\overline{1.01}$ & 0.315 & $\overline{\mathrm{NS}}$ \\
\hline
\end{tabular}

\section{DISCUSSION}

Esophageal variceal bleeding is a major cause of mortality in patients with portal hypertension. Endoscopic interventions, either by endoscopic variceal sclerotherapy or better by endoscopic variceal ligation are effective means of control of variceal bleeding [21]. Endoscopic intervention may be followed by ulcer formation, post injection sclerotherapy ulcer or post banding ulcer, that may be exacerbated by gastric acid. These ulcers may lead to further bleeding. Also endoscopic intervention is associated with annoying symptoms like chest pain, dysphagia and heart burn [5,12]. Most of these symptoms are also induced by acid. This study was designed to evaluate the role of rabeprazole as a member of the PPI group in prevention of post sclerotherapy ulcer formation, recurrent bleeding and reduction of the post injection symptoms.

Rabeprazole was selectively used in this study as it is little different in its metabolism, being converted more rapidly to the activated sulphenamide and also dissociated more rapidly from the $\mathrm{H}^{+} \mathrm{K}^{+}$ATPase, resulting in both a faster rate of inhibition and a shorter duration of action [22,24]. Rabeprazole is also the PPI less affected by the hepatic CYP2C19 metabolism [23,24]. Rabeprazole was used only for 2 months follow up period to avoid the development of long term use complications of PPIs like increased risk of bone fractures, anemia, and hypo-magnesemia and to limit the cost of the drug as much as possible.

All baseline demographic, clinical, laboratory, sonographic, endoscopic findings, symptoms after first EIS and the Child Pugh class had no statistically significant differences between the 2 groups. Moreover, in the present study there were no statistically significant differences regarding the $\mathrm{CBC}$ parameters of both groups during the 2 months follow up period. These results were in agreement with those reported by Dotan et al. [26] who evaluated 468 patients received pantoprazole and 468 controls for the development of thrombocytopenia and found no difference in the incidence of thrombocytopenia between both groups [25].

In this study there were no statistically significant differences between cases and controls regarding endoscopic findings during the follow up period, no statistically significant differences between the two groups in the incidence of sclerosant ulcer development. These results were in concordance with that reported by Shaheen et al. [27] as they evaluated 42 patients after EVL and randomization 
into placebo and pantoprazole treated groups (pantoprazole $40 \mathrm{mg}$ for 10 days). They found that subjects receiving pantoprazole after EVL had significantly smaller post-banding ulcers on follow-up endoscopy than subjects receiving placebo. However, the total ulcer number was not different between the groups [26]. Akahoshi et al. [28] also agree with the present study as they found that rabeprazole was associated with faster healing of post sclerotherapy ulcers [27].

In this study there was statistically highly significant decrease in all post injection adverse symptoms in the rabeprazole treated group throughout the follow up period. These results were in agreement with that reported by Akahoshi et al. [28] who compared the results of using rabeprazole $20 \mathrm{mg}$ once daily versus famotidine $20 \mathrm{mg}$ twice daily and found that the H2-blocker group experienced a significantly higher number of days of heartburn and dysphagia than did the PPI group. Finally, they stated that rabeprazole treatment prevents sclerotherapy-associated gastroesophageal reflux and improves the subjective symptoms following EIS [27]. On the other hand, Shaheen et al. [27] used pantoprazole for 10 days on 42 patients and found no significant symptoms improvement and Boo et al. [29] who used pantoprazole for 7 and 14 days and found no significant difference in symptoms [26,28]. This difference could be attributed to the use pantoprazole and the shorter duration of the two studies. Also, the two previously mentioned studies worked using band ligation which by nature has fewer symptoms than sclerotherapy.

In the present study, at the $2^{\text {nd }}$ and $6^{\text {th }}$ weeks of follow up, the incidence of moderate recurrent bleeding was significantly lower in the rabeprazole group, while the incidence of mild and severe recurrent bleeding and total cases with recurrent bleeding were not significantly affected. The total number of patients who developed recurrent bleeding at the end of the follow up period in the treated group was 7 versus 23 in the control group which was highly significantly lowered.

These results were in concordance with the studies conducted by Shaheen et al. [27] and Boo et al. [29], in their studies 3 patients and 2 patients bled from post banding ulcer respectively all were in placebo group. The larger number of recurrent bleeding in the present work may be due to larger sample size and longer follow up period and the fact that sclerotherapy is done on patients with active variceal bleeding unlike banding which is done usually for primary prophylaxis $[26,28]$.

At the end of our follow up period, there were no significant differences between both groups regarding the infectious or non infectious complications that can be induced by the use of rabeprazole. There was no significant difference regarding the development of fever, hepatic encephalopathy, SBP, diarrhea and chest infection. It was found that $32 \%$ of cases suffered from hepatic encephalopathy versus $26 \%$ of the controls. $14 \%$ of cases complained of one attack of chest infection versus $8 \%$ of controls and $2 \%$ of cases complained of 2 attacks of chest infection versus $0 \%$ of controls. $18 \%$ of PPI treated cases had one day of diarrhea versus $16 \%$ of controls, $14 \%$ of cases had 2 days of diarrhea versus $6 \%$ of controls, and $4 \%$ of cases had 3 days of diarrhea versus $2 \%$ of controls. $57.1 \%$ of the admitted cases who had diagnostic follow up ascitic fluid aspiration had developed SBP with TLC of the ascetic fluid equals to or greater than 400 versus $50 \%$ of the controls. Conversely, Gipiuliano et al. [30] and Johnstone et al. [31] found increased risk of community acquired pneumonia with the PPI use; in this study no significant difference between both groups in risk of development of pneumonia $[29,30]$. This difference may be due to short duration and single small dose of rabeprazole use in our study.

Deshpande et al. [32] and Howell et al. [33] found increased risk of colistridium difficile induced diarrhea associated with PPI use. However in this study no significant difference between both groups regarding diarrhea may be because not all cases of diarrhea with PPI use must be due to colistridium difficile infection, besides, in the present study the colistridium difficile toxin wasn't evaluated. Also Deshpande et al. [32] had used prophylactic antibiotics with PPI, which is also associated with increased risk of colistridium difficile diarrhea [31]. Howell et al. [33] used PPI daily or greater than daily dose and for longer duration than ours [32].

In this study there was no significant difference between both groups regarding the incidence of development of SBP, unlike the study conducted by Bajaj et al. [34] in which the incidence of development of SBP in PPI group was 30\% [33]. This difference may be due to larger population size, longer follow up period and concomitant 
use of antibiotics and the presence of a decompensated cirrhosis group in their study.

In this study no significant difference was found between the 2 groups regarding the development of hepatic encephalopathy, unlike the results recorded by Lin et al. [35] who found that patients with $\mathrm{HE}$ had a significantly higher rate of PPI use $(89.1 \%)$ compared with non-HE patients $(63.6 \%)$. The difference could be attributed to the larger sample size [34]. Also there were concomitant drug use as lactulose in the present work.

\section{CONCLUSION}

The use of rebeprazole at a dose of $20 \mathrm{mg}$ daily after sclerotherapy can help controlling the post sclerotherapy adverse symptoms and reduce the risk of recurrent bleeding. These effects are not reflected on the endoscopical findings. This beneficial effect is mediated without significant increase of the risk of chest, gastrointestinal or ascetic fluid infections and without increasing the rate of hepatic coma.

Ethical approval: Approved.

Funding: None.

Conflict of interest: None.

\section{REFERENCES}

1. De Francis R: Revising consensus in portal hypertension: Report of the Baveno V consensus workshop on methodology of diagnosis and therapy in portal hypertension. J Hepatol. 2010; 53: 762-8.

2. Villanueva C, Balanzo J, Novella MT, Soriano G, Sáinz S, Torras X et al : Nadolol plus isosorbidemononitrate compared with sclerotherapy for the prevention of variceal bleeding. $N$ Engi J Med 1996; 334: (25): 1624-9.

3. Lim EJ, Gow PJ and Angus PW: Endoscopic variceal ligation for primary prophylaxis of esophageal variceal hemorrhage in pre-liver transplant patients. Liver Transpl. 2009; 15(11): 1508-13.

4. Lo GH, Lai KH, Cheng JS, Lin CK, Huang JS, Hsu PI et al.: Emergency banding ligation versus sclerotherapy for the control of active bleeding from esophageal varices. Hepatology 1997; 25: $1101-4$
5. Young MF, Sanowski RA and Rasche R: Comparison and characterization of ulcerations induced by endoscopic ligation of esophageal varices versus endoscopic sclerotherapy. Gastrointest Endosc.1993;39: 119-122.

6. Bardou M, Martin J and Barkun A: Intravenous proton pump inhibitors : an evidence-based review of their use in gastrointestinal disorders. Drugs. 2009; 69(4), 435-448.

7. Baker DE: Intravenous proton pump inhibitors. Rev. Gastroenterol. Disord.2006;6(1), 22-34.

8. Wallace JL, Sharkey KA: Pharmacotherapy of gastric acidity, peptic ulcers, and gastroesophageal reflux disease. In: Perils and Pitfalls of Long-term Effects of Proton Pump Inhibitors. Expert Rev Clin Pharmacol.2011; 6 (4):443-451.

9. Goldstein JA: Clinical relevance of genetic polymorphisms in the human CYP2C subfamily. Br. J. Clin. Pharmacol.2001; 52: 349-55.

10. Tytgat GN: Shortcomings of the first-generation proton pump inhibitors. Eur. J. Gastroenterol. Hepatol. 2001; 13(1): 29-33.

11. Hou MC, Lin HC, Lee FY, Chang FY, Lee SD: Recurrence of esophageal varices following endoscopic treatment and its impact on rebleeding: comparison of sclerotherapy and ligation. J Hepatol.2000;32: 202-208.

12. Hidaka H, Nakazawa T, Wang G, Kokubu S, Minamino $\mathrm{T}$, Takada $\mathrm{J}$ et al : Long-term administration of PPI reduces treatment failures after esophageal variceal band ligation: a randomized, controlled trial. J Gastroenterol Hepatol. 2013; 28(4):684-9.

13. Marks RD, Arnold MD and Baron TH: Gross and microscopic findings in the human esophagus after esophageal variceal band ligation: a postmortem analysis. Am J Gastroenterol.1993; 88: 272-274.

14. Child CG and Turcotte JG: Surgery and portal hypertension. In: The liver and portal hypertension. Major ProblClin Surg.1964;1:1-85.

15. Cales P, Oberti F, Bernard B, Payen JL: Evaluation of Baveno recommendations for grading of Oesophageal varices .J Hepatol. 2003; 39(4) : 657-9

16. Ali N and Sumaira M: Portal Hypertension. $J$. Gastrointestinal radiol.2002, 23:234.

17. Thakeb F, Salem SA, Abdallah M, el Batanouny M: Endoscopic diagnosis of gastric varices. Endoscopy. 1994 Mar; 26(3):287-91

18. Carpinelli L, Primignani M, Preatoni P, Angeli P, Battaglia G, Beretta L, et al: Portal hypertensive gastropathy: reproducibility of a classification, prevalence of elementary lesions, sensitivity and specificity in the diagnosis of cirrhosis of the liver. A NIEC multicentre study. New Italian Endoscopic Club. Ital J Gastroenterol Hepatol. 1997; 29(6):533-40. 
19. Cappell MS and Friedel D: Initial Management of Acute upper Gastrointestinal Bleeding: From Initial Evaluation up to Gastrointestinal Endoscopy. Med Clin N Am.2008;92:491-509.

20. Garcia-Tsao G, Sanyal AJ, Grace ND, Carey W: Practice Guidelines Committee of the American Association for the Study of Liver Diseases; Practice Parameters Committee of the American College of Gastroenterology: Prevention and management of gastroesophageal varices and variceal hemorrhage in cirrhosis. Hepatology. 2007;46(3):922-38

21. Del Olamo IA, Pena A, Serra MA,Wassel AH, Benages A, Rodrigo JM: Predictors of morbidity and mortality after the first episode of upper gastrointestinal bleeding in liver cirrhosis. J Heopatol.2000;32 (1): 19-24.

22. Garcia-Tsao G, Bosch J: Management of Varices and Variceal Hemorrhage in Cirrhosis. $N$ Engl $J$ Med.2010;362:823-32.

23. Besancon M, Simon A, Sachs G, Shin JM:Sites of reaction of the gastric $\mathrm{H}$, K-ATPase with extracytoplasmicthiol reagents. $J$ Biol Chem. 1997; 272: 22 438-46.

24. Williams MP, Sercombe J, Hamilton MI, Pounder RE: A placebo-controlled trial of the effects of 8 days of dosing with rabeprazole versus omeprazole on 24-h intragastric acidity and plasma gastrin concentrations in young healthy male subjects. Aliment Pharmacol Ther. 1998; 12: 107989.

25. Robinson M: Review article: the pharmacokinetics of proton pump inhibitors - overview and clinical implications. Aliment. Pharmacol. Ther.2004;20: (6): $1-10$.

26. Dotan E, Katz R, Bratcher J, Wasserman C, Liebman M, Panagopoulos $\mathrm{G}$ et al: The prevalence of pantoprozole associated thrombocytopenia in a community hospital .Expert Opin. Pharmacother. 2007;8(13):2025-2028.

27. Shaheen NJ, Stuart E, Schmitz SM, Mitchell KL, Fried MW, Zacks S, et al: pantoprazole reduces the size of post-banding ulcers after variceal band ligation: a randomized, controlled trial.Hepatology. 2005;41(3):588-94.
28. Akahoshi T, Kawanaka H, Tomikawa M, Saeki H, Uchiyama H, Ikeda Tet al: The effect of proton pump inhibitor (PPI : Rabeprazole) on reflux esophagitis after endoscopic injection sclerotherapy (EIS), a randomized control study (24 hour-pH monitoring). Fukuoka Igaku Zasshi. 2013; 104(12):483-9.

29. Boo GB, Oh JC, Lee BJ, Lee DM, Kim YD, Park $\mathrm{CG}$, et al: The effect of proton pump inhibitor on healing of post-esophageal variceal ligation ulcers. Korean J Gastroenterol. 2008 ; 51(4): 232-40.

30. Gipiuliano C, Wilhelm SM, Kale-Pradhan PB: Are proton pump inhibitors associated with the development of community-acquired pneumonia? A meta-analysis. Expert Rev. Clin. Pharmacol. 2012; 5(3), 337-344.

31. Johnstone J, Nerenberg K. Loetb M: Metaanalysis: proton pump inhibitor use and the risk of community acquired pneumonia. Aliment. Pharmacol Ther.2010;31 (11). 116 5-117 7.

32. Deshpande A, Pant C, Pasupuleti V, Rolston DD, Jain A, Deshpande N, et al: Association between proton pump inhibitor therapy and Clostridium difficile infection in a meta-analysis. Clin. Gastroenterol. Hepatol.2012;10(3), 225233.

33. Howell MD, Noigck V, Grgurtch P, Soulliard $\mathrm{D}$, Novack L, Pencina $\mathrm{M}$ et al: Iatrogenic gastric acid suppression and the risk of nosocomial Colstridium diffficile infection. Arch. Intern. Med.2010;170(9), 784-790.

34. Bajaj JS, Zadvornova Y, Heuman DM, Hafeezullah M, Hoffmann RG, Sanyal AJ et al: Association of proton pump inhibitor therapy with spontaneous bacterial peritonitis in cirrhotic patients with ascites. Am J Gastroenterol. 2009; 104(5) : 1130-4

35. Lin ZN, Zuo YQ, Hu P: Association of Proton Pump Inhibitor Therapy with Hepatic Encephalopathy in Hepatitis B Virus-related Acute-onChronic Liver Failure.Hepat Mon. 2014; 7; 14(4):e16258.

Peer reviewer: Abeer Nafee, Professor of Tropical Medicine and Hepatogastroenterology, Faculty of Medicine, Zagazig University, Egypt.

Editor: Tarik Zaher, Professor of Tropical Medicine and Hepatogastroenterology, Faculty of Medicine, Zagazig University, Egypt 